\title{
Rapidly Emerging Megaureteral Stump Empyema after Pretransplant Nephrectomy: A Case Report
}

\author{
Rabea Ahmed Gadelkareem \\ Assiut Urology and Nephrology Hospital, Faculty of Medicine, Assiut University, Assiut, Egypt
}

\section{Keywords}

Empyema - Megaureter · Pretransplant nephrectomy ·

Reflux · Ureteral stump

\begin{abstract}
Acute presentation of ureteral stump empyema after pretransplant nephrectomy has not been reported so far. We report on a 25 -year-old male patient with end-stage renal disease due to congenital vesicoureteral reflux and a longterm history of management of infected kidneys including the last step of pretransplant right native nephrectomy. Within 2 weeks after operation, he presented with clinically visible ureteral stump empyema. Computed tomography and surgical exploration confirmed the diagnosis of a huge retentive stump of obstructive-refluxing megaureter which was excised with a smooth postoperative course. So, ureteral stump empyema may develop after a pretransplant nephrectomy and threaten the transplant process and the patient's life. Consecutive nephrectomy and ureterectomy procedures to keep theiliac fossa fresh for kidney transplantation do not outweigh the risks of ureteral stump infections. Therefore, simultaneous nephroureterectomy is mandatory in the case of obstructive-refluxing megaureter.

(c) 2018 The Author(s)

Published by S. Karger AG, Basel
\end{abstract}

\section{Introduction}

Pretransplant nephrectomy is a surgical step in the preparation for kidney transplantation. It is advisable for many reasons [1]. However, ureteral stump complications may develop due to vesicoureteral reflux disease (VURD), infections [2], and retained stones [3]. When a constellation of symptoms including fever, lower abdominal pain, irritative lower urinary tract symptoms, and hematuria results from the ureteral stump, it is known as the "ureteral stump syndrome" [4]. Moreover, suppuration may develop forming a rare variant known as the "ureteral stump empyema" [5-7]. The question is whether preserving the potential site of kidney transplantation surgically fresh deserves leaving the ureteral stump for a later session.

\section{Case Report}

A 25-year-old male patient had chronic kidney disease and presented to our hospital seeking for kidney transplantation. The patient's history, physical examination, and old follow-up file referred to long-term management in other centers for congenital VURD. Both kidneys were infected, while the right one was pyonephrotic and was drained by percutaneous nephrostomy to control the infection and progression of the chronic kidney disease. Also, the left kidney had a history of repeated JJ stent insertions,

\begin{tabular}{ll}
\hline KARGER & $\begin{array}{l}\text { ( } 2018 \text { The Author(s) } \\
\text { Published by S. Karger AG, Basel }\end{array}$ \\
E-Mail karger@karger.com & $\begin{array}{l}\text { This is an Open Access article licensed under the Creative Commons } \\
\text { Attribution-NonCommercial-4.0 International License (CC BY-NC) } \\
\text { (http://www.karger.com/Services/OpenAccessLicense), applicable to } \\
\text { the online version of the article only. Usage and distribution for } \\
\text { commercial purposes requires written permission. }\end{array}$
\end{tabular}

Rabea A. Gadelkareem

Assiut University

Elgamaa Street

Assiut 71515 (Egypt)

E-Mail dr.rabeagad@yahoo.com 


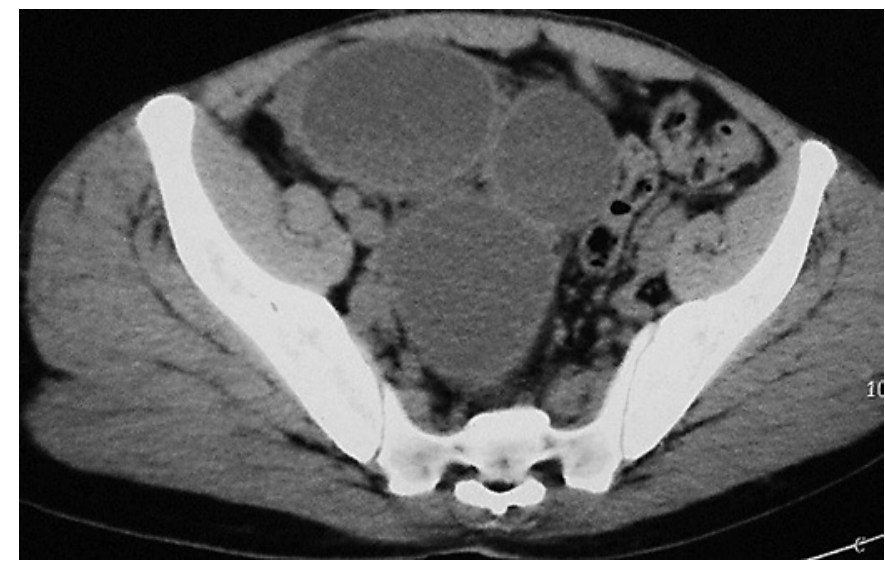

Fig. 1. Noncontrast computed tomography, transverse section: right-sided folded ureteral stump with contact between the anterior and posterior abdominopelvic walls. Notice the pushed peritoneum and its contents to the left side.

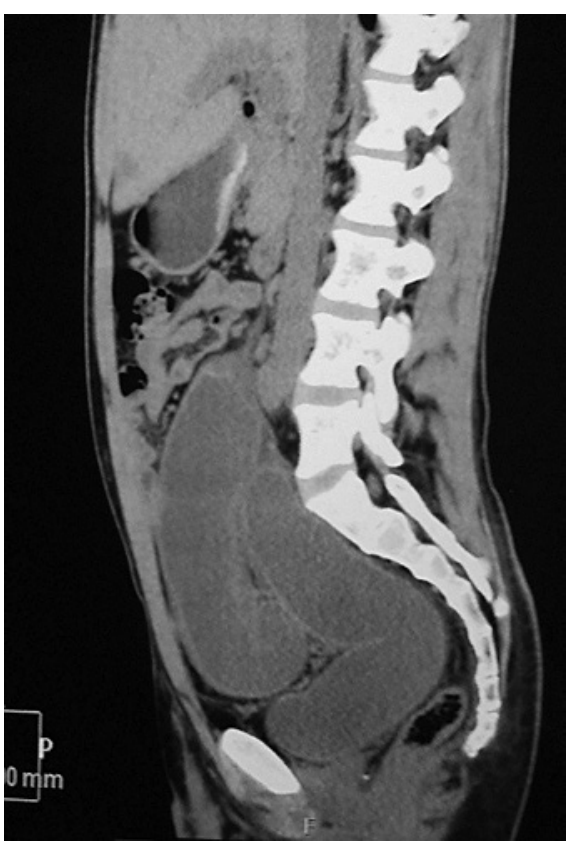

Fig. 2. Noncontrast computed tomography, sagittal section: intercommunicating folded fluid-filled loop of the tortuous ureteral stump. Longitudinal view of the returning folds of the megaureter.

but at presentation it was atrophied without manifestations of gross infections. Several courses of antimicrobial therapy were tried according to culture and sensitivity tests. Also, supportive measures including diet regulations and supplementations including oral calcium and subcutaneous erythropoietin have been described regularly. However, end-stage renal disease (ESRD) developed and the patient has been maintained on regular hemodialysis through an arteriovenous fistula for the last 4 months with reduced urine output at presentation. As a preparation for kidney trans- plantation, pretransplant native nephrectomies were scheduled to eradicate the risks of infections. To avoid an anephric state, laparoscopic left native nephrectomy was scheduled after the management of the right side. Firstly, open right nephrectomy was done through a flank incision because of the highly suspected extensive adhesions of pyonephrosis and long-standing percutaneous nephrostomy. The ureter was markedly dilated and tortuous where it was evacuated by suction and ligated at a level above the iliac vessels to avoid adhesions in this potential site for transplantation and keep it in a surgically fresh state. The right ureteral stump was left for spontaneous drainage through the urinary bladder depending on its proposed refluxing state in the established diagnosis of VURD. The postoperative course was uneventful, and the patient was discharged on the 5 th postoperative day.

On the 14th day after surgery, the patient presented urgently with a progressively increasing right lower quadrant abdominal pain and swelling with fever for 4 days. On physical examination, he looked toxemic and uremic. His blood pressure was $110 / 70 \mathrm{~mm}$ $\mathrm{Hg}$, his pulse was 84 beats/min, and his temperature was $38.5^{\circ} \mathrm{C}$. There was a visible and tender smooth surface mass in the right iliac region extending up to the level of the umbilicus. Signs of generalized peritonitis were absent. Values of the laboratory workups for infection were reported including markedly elevated C-reactive protein level $(109 \mathrm{mg} / \mathrm{L})$ and count of white blood cells in the blood picture $\left(18.4 \times 10^{3} / \mathrm{mm}^{3}\right)$. Other completed laboratory workups including serum creatinine level of $4.5 \mathrm{mg} / \mathrm{dL}$ and hemoglobin level of $9.8 \mathrm{~g} / \mathrm{dL}$ were acceptable for the patient's state. Urgent abdominal ultrasonography and noncontrast computed tomography scanning revealed a huge multilocular cystic mass occupying most of the right retroperitoneal space down to the urinary bladder with thick fluid contents (Fig. 1, 2). However, the surgical bed of the removed kidney contained a small hematoma of $2 \times 2 \mathrm{~cm}$. Owing to the known medical and surgical history of the patient, rapid course formation of this mass, and the hemodynamic stability, a retentive ureteral stump was suspected. As an initial management, a urethral catheter was inserted, intravenous third-generation cephalosporin agent and antipyretic were given, and the patient was observed for a few hours. However, the swelling and fever did not resolve.

Urgent laparotomy through a midline suprapubic incision was performed. It revealed a massively dilated, tortuous, folded, and tense right ureteral stump extending from a point a few centimeters above the iliac vessels to the urinary bladder. Tissue edema and adhesions were significant, but the stump was freed and dissected from the surroundings. The juxtavesical part was noticed to be very narrow, which referred to a misdiagnosed combined obstructed-refluxing megaureter. Moreover, with surgical manipulations, there was a sudden release of about $1.5 \mathrm{~L}$ of heavily turbid grayish fluid through the urethral catheter. Dissection was completed and a transfixing ligature of the ureterovesical junction was taken and followed by excision of the stump which contained another $1.5 \mathrm{~L}$ of more thick mud-like deposits. Hemostasis and wound closure were done on a tubal drain.

The postoperative course was smooth with dramatic improvement of symptoms and laboratory parameters. Culture and sensitivity tests of the drained suppuration revealed Escherichia coli, which was treated accordingly by a complementary course of a third-generation cephalosporin agent with continuation of the supportive supplements and measures. Follow-up by abdominal ultrasonography for several months revealed no further collections in the surgical beds or the entire abdomen. 


\section{Discussion}

Ureteral stump empyema is an uncommon variant of ureteral stump syndrome $[6,7]$. It evolves from retained pus in an obstructed stump [3]. It is important to treat an ureteral stump in the pretransplant period as a preparation for kidney transplantation and eradication of infections, which is the most common indication in these cases [1]. The current case is unique in many aspects which may deserve discussion: it was rapidly emerging, of a huge size, with possibly an underlying missing retentive mechanism, and occurred in a pretransplant ESRD patient.

The natural history of development of ureteral stump empyema after nephrectomy is usually of long-term duration extending up to 20 years $[3,6]$. Surprisingly, the current case of empyema presented within days after nephrectomy. This could be attributed to the missed nature of the underlying obstructed-refluxing megaureter, previous pyonephrosis, and the ESRD stage.

Androulakakis et al. [4] reported that the long tortuous and atonic stump with a narrow vesicoureteral junction is mimicking a retentive diverticulum. It may rely upon the "pressure sink" effect [8]. Here, in concordance, massive dilatation and long-standing infection of the ureter might make it atonic. The residual stump was roomy, and the narrow junction acted as a one-way valve. On voiding, it allowed the passage of urine from the highpressure compartment of the urinary bladder to the lowpressure one of the stump and not the opposite. This process was proposed to be continued until the stump became tense enough to resist this effect at the narrow junction segment. Also, the obstruction of this narrow junction was potentiated by the old thick suppuration deposits. Intraoperative release of the suppurative contents through the urethral catheter referred to the patency of this narrow vesicoureteral junction: the obstructed-refluxing megaureter.

In spite of the cases which have been described or illustrated as massively dilated and refluxing $[9,10]$, the current case has been the first time that the stump mass becomes clinically visible and retains large amounts of pus. Initially, it was confused with other differential diagnoses such as secondary retroperitoneal hemorrhage and intestinal obstruction. However, the known surgical history, suspicious clinical signs, and imaging facilitated the diagnosis of megaureteral stump empyema.

In spite of the emergence of minimally invasive techniques for the management of ureteral stumps $[2,6]$, urgent laparotomy and radical excision were performed to avoid possibilities of repeated scenarios which may im-

Pretransplant Megaureteral Stump

Empyema pede infection eradication and, in turn, planned kidney transplantation. In spite of the near surgeries within 14 days, judicious management saved this patient from these life-threatening risks. His potential related living kidney donor was his mother who, however, developed diabetes mellitus during this stage of preparation. Although this young adult patient seemed to be unlucky to have lost his related potential kidney donor and had to wait for another donor, his chances for kidney transplantation are still viable after the resolution of the offending risks.

Here, a unique pretransplant urological case scenario was presented. In the current case, maintenance hemodialysis with a reduction of the urine output obscured the original picture of the ureters and kidneys. They should be studied meticulously by imaging to reveal the underlying nature of obstruction. So, the ureteral stump of an infected kidney must be removed with the native kidney down to the urinary bladder securing the vesicoureteral junction to ameliorate the potential risks of infections. It is impossible to advance to kidney transplantation before the complete eradication of infections [1]. Although VURD was bilateral with an old history of infections, native nephrectomies were scheduled in stages in our case. Simultaneous bilateral native nephrectomies were a viable option to avoid the inconveniences of more surgeries. However, it is our center's policy to avoid an anephric state whenever possible. Thus, the silent state of the left kidney promoted the decision to keep it, temporarily, for the time being and preserve its proposed regulatory residual hormonal and urine secretion functions until having found a suitable donor. Once a suitable donor has been found, the left iliac fossa is still surgically fresh, which is our proposed site for kidney transplantation.

In conclusion, pretransplant nephrectomy in ESRD patients with pyonephrosis and VURD is a challenging surgery and all the possible precautions should be considered. To decide simultaneous or consecutive pretransplant native nephrectomy and ureterectomy, meticulous evaluation of the distal ureter is mandatory before advancing to kidney transplantation. However, simultaneous surgery is preferred for the eradication of infections. Moreover, obstructive or obstructive-refluxing megaureter with an infected kidney is recommended as an absolute indication for simultaneous pretransplant native nephroureterectomy. Keeping the iliac fossa surgically fresh for the time of kidney transplantation does not outweigh the potential life-threatening risks and complications of the ureteral stump in cases of infected kidneys. 


\section{Statement of Ethics}

A written consent was obtained from the patient.

\section{Disclosure Statement}

The author has no conflicts of interest to declare.

\section{References}

1 Shoma AM, Eraky I, El-Kappany HA. Pretransplant native nephrectomy in patients with end-stage renal failure: assessment of the role of laparoscopy. Urology. 2003 May;61(5): 915-20.

2 Alenezi H, Eltiraifi AE, Alomar M. Minimally invasive surgery for the treatment of ureteral stump syndrome. Urol Ann. 2015 Oct-Dec; 7(4):454-8.

3 Prajsner A, Szewczyk W, Gawron R. Empyema of retained ureteral stump with gross stone 20 years after nephrectomy. Urology. 2009 May;73(5):993-4.
4 Androulakakis PA, Stephanidis A, Antoniou A, Christophoridis C. Outcome of the distal ureteric stump after (hemi)nephrectomy and subtotal ureterectomy for reflux or obstruction. BJU Int. 2001 Oct;88(6):586-9.

5 Hyman A. Empyema of the ureteral stump following incomplete ureterectomy. Ann Surg. 1923 Sep;78(3):387-97.

6 Ikeda D, Matsutani R, Fukuda M, Fuse H, Hirano $S$. Transurethral fulguration for empyema of ectopic ureteral stump. Int J Urol. 2003 Dec;10(12):664-6.
7 Labanaris AP, Zugor V, Smiszek R, Nützel R, Kühn R. Empyema of the ureteral stump. An unusual complication following nephrectomy. ScientificWorldJournal. 2010 Mar; 10: 380-3.

8 Wilson B, Klufio G. The radiological and urodynamic significance of large bladder diverticula. Clin Radiol. 1985 Sep;36(5):521-4.

9 Memon MA, Neal PM, Patterson PJ. Massively dilated and refluxing ureteral stump. Infect Urol. 1998;11(4):101-3.

10 Pereira J, Reis A, Castro R. [Ureteral stump empyema after heminephrectomy]. Acta Med Port. 2014 May-Jun;27(3):404. 